\title{
'UNREALART'. A NEW MEDIUM FOR ARTISTIC EXPRESSION USING A COMMERCIAL GAME ENGINE: GALLERIES AND INSTALLATIONS.
}

Colin B. Price

Computing

University of Worcester

Henwick Grove

Worcester, WR2 6AJ

United Kingdom

c.price@worc.ac.uk

http://www.worc.ac.uk/departs/bm_it/colin/Resources/EVA2008

\begin{abstract}
In this paper, we explore the use of a commercial computer game-engine "Unreal Tournament 2004" (UT2004) to produce "Immersive Environments" (IEs) which provide the digital artist with a new mode of expression, to engage with the viewing public as individuals or in collaboration. We explore two modalities of Art, the 'gallery' and the 'installation'. Through a reflection on various twentieth-century abstract artists, we indicate how this technology may be engaged to more fully realize their philosophies and projects. Finally, we suggest how this technology may lead to new forms of artistic expression in our contemporary digital world.
\end{abstract}

\section{INTRODUCTION}

Art has become a fundamental, even primordial conversation, which listens to all aspects of contemporary life, from science, to the political agenda and beyond, into the spiritual. Within this conversation, Art speaks and therefore informs; it is generative, it makes a statement. Conversations occur within a socio-cultural context, that domain of agendas which are situated within our academic and commercial worlds. These conversations must use the contemporary vocabulary of expression, defined by the current modi of technology and thought. In this paper we address one emergent technology and philosophy, computer games, and suggest that this may shift the paradigm of artistic expression into a new epoch. Through reflection on the works and motivations of selected twentieth-century artists, we identify concepts and styles which may be realized within computer game technology. We do not construct 'games', but rather 'Immersive Environments' (IEs) where the interplay between the creative Artist and the individual or collaborative 'viewer' may be realised.

UT2004 has a sophisticated editing tool, "UnrealEd" which allows the production of rooms and geometrical content, specification of lighting and movement of objects and programming custom behaviour in the language "Unreal Script". In this paper we mention how the various elements of UnrealEd may be used to construct art.

This paper is structured as follows. In Section 2 we explore the works of significant twentieth-century artists, and propose how their artworks may inform a new generation of 'Gallery' experiences. Section 3 discusses 'Installation Art'. Our own perspective is crystallized in Section 4 which summarises our theories and experiments in interactive dynamic art. The various concepts discussed (such as dynamics, color space) are collated in Section 5. An associated web-site available at the above URL contains all the IEs discussed below, and instructions how to install the software. 


\section{UNREALGALLERY: EXPLORATIONS IN $20^{\text {th }}$-CENTURY ART} Robert Delaunay

During 1912-1913 Delaunay developed a style of painting which achieved a dynamic based on color contrasts and harmonies. He said "But what is of great importance to me is the observation of the movement of colors [1]. He identified the concept of movement being produced by "odd elements" in a reality of "vastness", where "simultaneity in light is harmony, the rhythm of colors" [2]. The IE shown in Figure 1 attempts to convey an impression of balanced floating forms without the grounding influence of gravity. These have been constructed using UnrealEd's 'intersect' and 'de-intersect' tools.

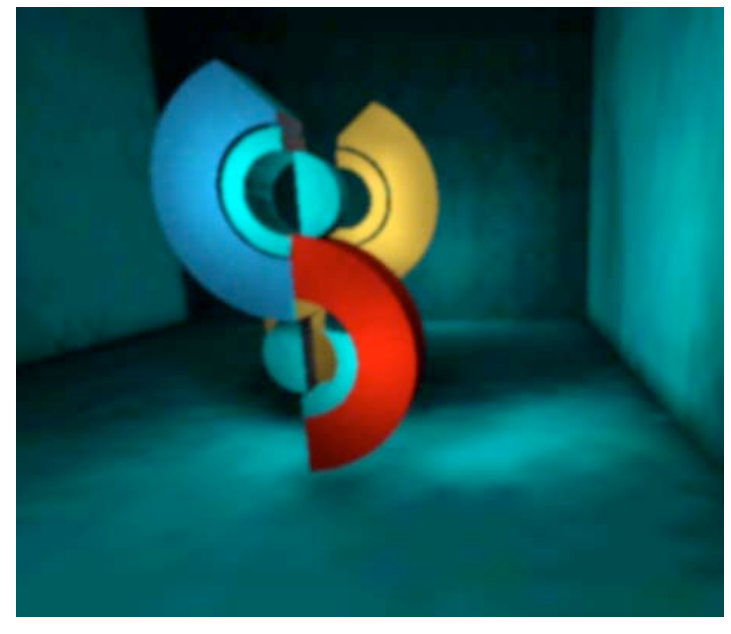

Figure 1. Robert Delaunay IE.

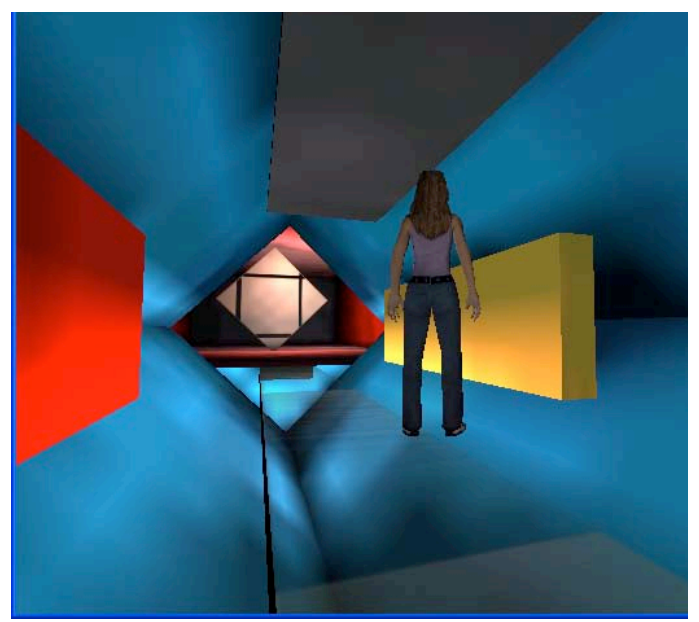

Figure 2. Piet Mondriaan 'lozenge’ IE.

\section{Piet Mondriaan}

Mondriaan's lozenge paintings (e.g. Composition 1A, 1990) challenge the vertical-horizontal aspect of our world as realized by structures such as buildings and roads located on the spherical planet, but experienced as this aspect in our localized place. This perspective is deconstructed in his 'lozenges' which rotationally displace our perspective by ninety degrees, While the localized framework is preserved, it is challenged; who can walk on a ninety degree gradient without feeling displaced? In Figure 2 our IE contains the flat Composition $1 A$ and extrudes the base horizontal stripe into a walkway. Moving into a lozenge-shaped corridor, the viewer is invited to experience a surrealistic environment while being grounded in. Wall and ceiling sheets have been placed to provide a perceptual framework of questioning the juxtaposition between the local geometry of our experience and to transcend this. Yet in some way this challenges the 'Mondrian Project'. To quote Mondriaan, "This attitude of the cubists to the representation of volume was contrary to my conception of abstraction which is based on the belief that this very space has to be destroyed. As a consequence, I came to the destruction of volume by the use of the plane" [3].

So Mondrian was led to destroy depth and space, and perhaps even light. Yet his spiritualism and desire to express a 'dynamic movement in equilibrium' encourages 
us to a reconstruct the third dimension. While Mondriaan deconstructs, we reconstruct, establishing, in the third dimension a metaphysical dimension of progression or ascendance. While our IE preserves the planar Mondriaan, our third dimension realizes the spiritual. After all, Mondriaan's lines become as thick as planes [4]. A similar reconstruction was achieved in the architecture of 'De Stijl' as exemplified by Gerrit Rietveld's Shroder House.

\section{Mark Rothko}

The culmination of Rothko's art is found in his 1949 paintings of large, ragged, engulfing and luminous canvases of few colors. While Newman had been concerned with making the spectator 'present', Rothko was interested in a more intimate involvement; the role of the viewer was to know that "a picture lives by companionship" [5]. A screenshot of one of our 'Rothko-Rooms' is shown in Figure 3 His painting No. 10 (1950) has been deconstructed and reconstructed, with front and back-lighting to generate a sense of luminous shapes floating in space. The viewer starts off observing the artwork from afar, and may approach and enter the ragged volume of color. Like our Newman canvases, these paintings are large. As Rothko said, "To paint a small canvas is to paint yourself outside your experience...". The scale of our IE, and of the canvas have been chosen to correctly reproduce the scale of the actual painting. Lighting has been set with attention to detail using UnrealEd.

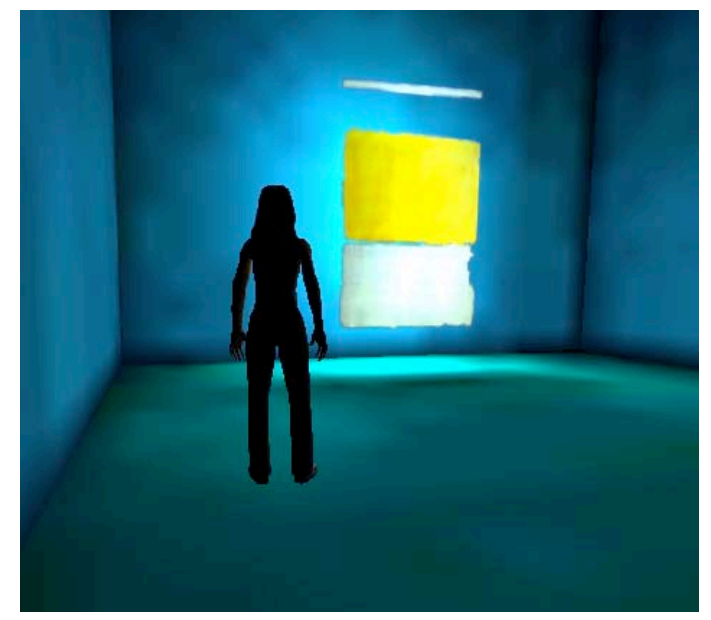

Figure 3. Mark Rothko No 10

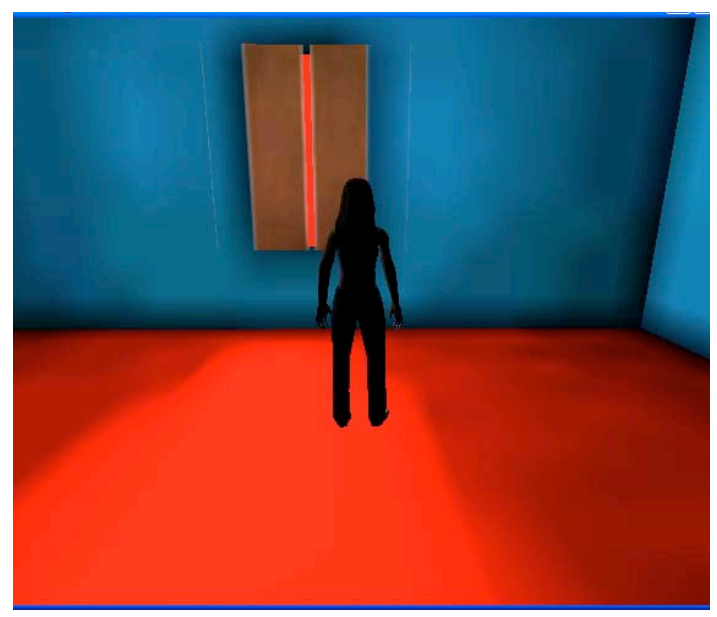

Figure 4. Barnett Newman Onement 1

\section{Barnett Newman}

Newman achieved his mature style in the late 1940's, delineating compositional areas on his canvas by the use of masking tape to obtain a narrow vertical divide. While painting Onement 1 he decided, on instinct, to color the divide with orange. "Suddenly, I realized that I had been emptying space instead of filling it" [6]. Such contrasting divides, (know as 'zips'), were to become a defining element of his style. Newman had turned to the Judaic tradition, in particular that wonderful combination of logic and mysticism, the Kabbalah where Adam is the divine light which emanates from God into the primeval vacuum. We read Newman's 'zips' as windows through which this light is 
both visible and illuminates. Figure 4 shows an IE where Onement 1 has been constructed with a radiating light source projecting into the gallery room. Newman's use of enormous scale, invoking the sublime, is achieved within UT, quite simply by construction of large gallery rooms, containing large canvases in relation to the scale of the viewer. Our IEs invoke a perception of scale and the sublime. Incorporation of UT lighting, and modification of light 'properties' (such as hue, light radius and 'cone') provide the artist with a palette of 'parameters' with which they can establish a visual experience.

\section{Bridget Riley}

Optical art explores the fallacies of the human visual system (HVS), and deals in a significant way with optical illusions which trick the viewer into constructing a percept of the artwork which transcends the actual canvas. Often it asserts some ambiguity. Riley's canvases such as Loss (1964) invoke a perception of depth and movement through the distortion of a repeated motif placed on a flat canvas. We do the opposite, paint an area with homogeneous motif, but on a surface which is not flat. This is done using UnrealEd's terrain facility which is more usually employed to create landscapes. Figure 5 Illustrates our IEs constructed in homage to Riley. The deformations of the plane have been obtained by programming mathematical functions using 'Matlab'. The surface shown here is a rendering of the mathematical function

$$
\left(1-e^{-\alpha x^{2}}\right) /\left(1-e^{-\alpha}\right) \text { where } x \text { is horizontal in space and } \alpha \text { is a parameter. }
$$

It is interesting to compare the perceptual effects of Riley's 'Optical-Art' with our mathematical deformed space. While both may invoke a vivid sense of movement, the appearance is different. Riley has produced an illusion, we have produced reality. The observed distortion of the circle motifs is a true depiction of distorted physical space: spheres placed on this surface will roll.

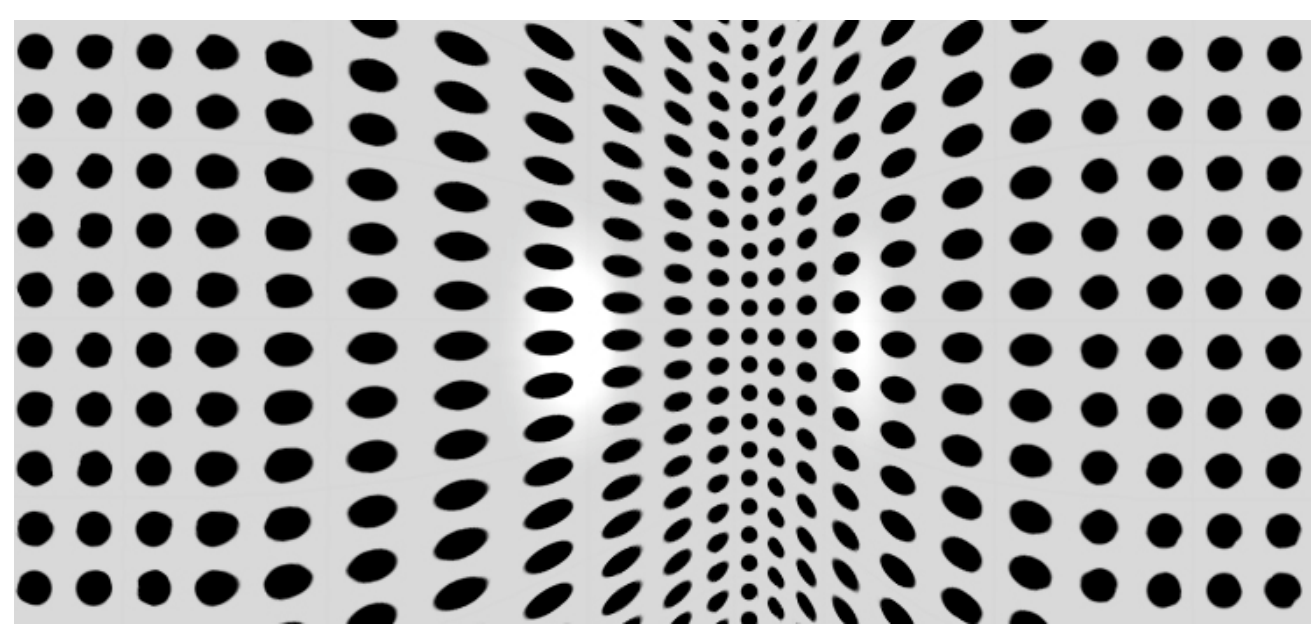

Figure 5. Homage to Bridget Riley. The illusion of depth and motion is no illusion. A homogeneous texture has been applied to a deformed plane. 


\section{UNREALINSTALLATIONS: $20^{\text {th }}$-CENTURY LOCATIVE ART}

\section{El-Lissitsky}

A primary exponent of the use of materials in a non-symbolic or literal mode is El-Lissitsky (1890-1941). In his Proun Rooms exhibited at the 1923 Berlin Art Exhibition, he constructed an installation designed to draw the viewers in and lead them through a programmed sequences of visual events. His concept of space was active and social, the need for circulation was fundamental. This space deconstructed the Renaissance insistence on perspective, which located each viewer in a constrained place. Space is no longer an abstract of the picture but a place where each must act. This space of Prouns was not a container for embellishment, but an arena demanding action from the viewers. One room from the IE is shown in Figure 6, which reflects ElLissitsky's 1923 Proun Room. Our IE includes "Non-Player Characters" (NPC's) which have been programmed to traverse the Proun Room as El-Lissitsky desired. Spoken messages triggered by UnrealEd's 'proximity triggers' can also guide the viewer through the room. The geometrical elements of the prouns are easily fashioned in UnrealEd.

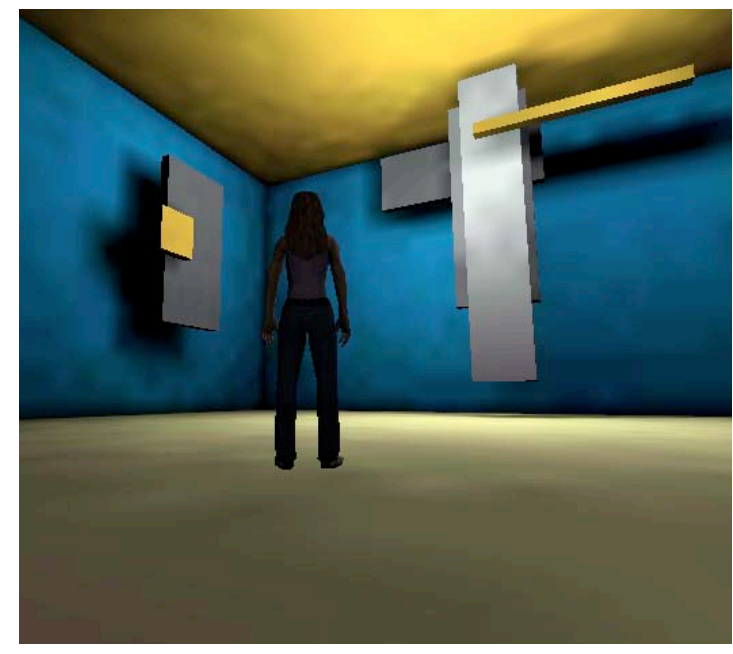

Figure 6. Reconstruction of El-Lissitsky's proun room as kinetic art.

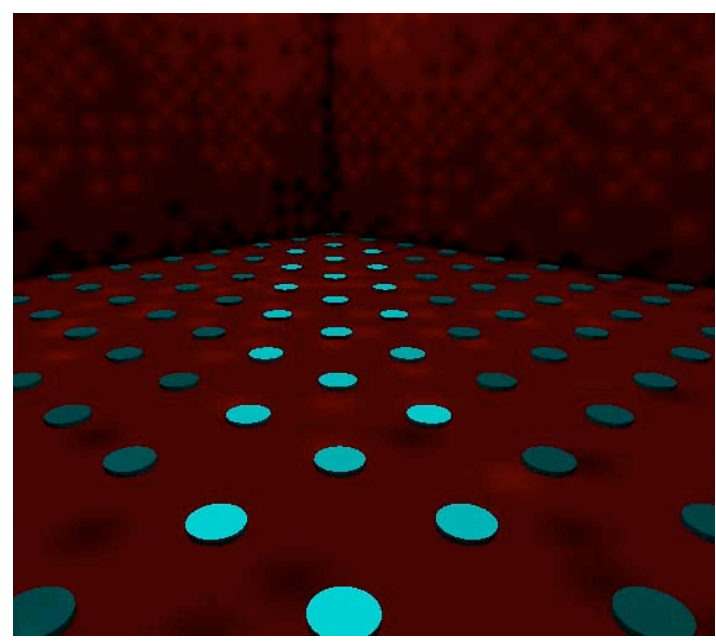

Figure 7. Coloured patches show the path of the previous viewers.

\section{Angela Bulloch}

Angela Bulloch in her multi-media Installations captures the affordances of our IEs and motivated their development. She combines programmable light, sound, video and text to explore both historical and digital Modernism. Her minimalist 'pixel boxes' are programmed with a dynamics driven by viewer presence. While existing in space, these boxes explore the meaning of conceptual space, where their interconnectivity demands the cognition of the visual relationships between color and rhythm. In her piece Fundamental Discord:16 (2005) she spreads sixteen pixel boxes on a four-square grid upright on the floor. We have developed a similar IE using luminous floor tiles, yet while Bulloch's pixel boxes hinder the circulation of the viewers, our space allow the viewer to interact with the tiles and change their luminosity. When the viewers step on a tile, its luminosity increases, then decays according to a time-constant. This results in 
the viewer's path being temporarily cast into space; the next viewers are attracted to this luminous path, which self-reinforces, Figure 7. While Bulloch resists all 'linguistic' interpretations of her work, asserting that meanings exist only in terms of relationships, we assert the mathematical 'language' is of 'Complex Dynamic Systems' (CDS) and 'Self Organization', which defines a formal, mathematical, but creative language of interactions (see Section 4). In a second piece, we have reconstructed Bulloch's 'pixel boxes' within our IE and have programmed (using Unreal Script) their state to respond dynamically to viewer interaction. This casts the viewer as an 'investigator', to observe, interact, experiment and so construct theories of the underlying dynamics, (see Figure 8).

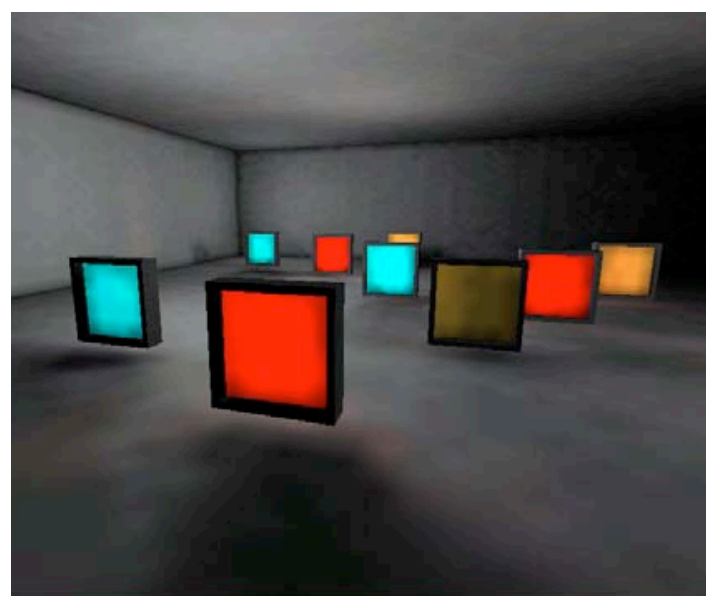

Figure 8. Bulloch's pixel boxes which respond to the viewer with light and sound.

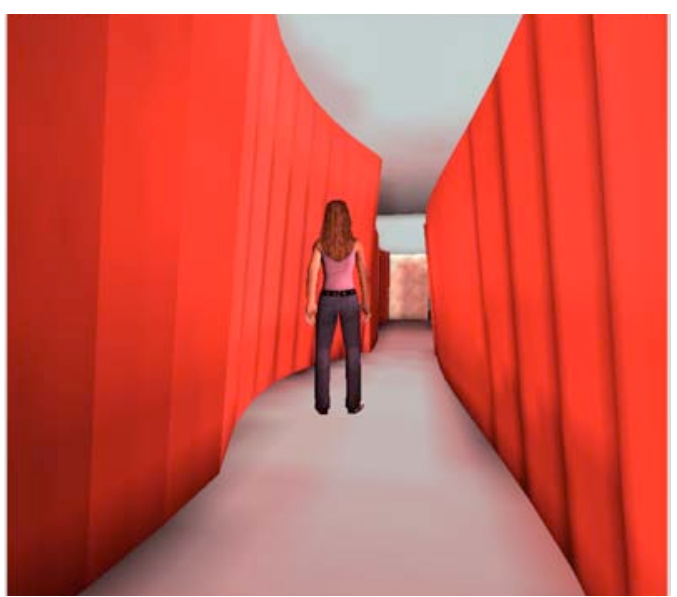

Figure 9. Richard Serra installation

\section{Richard Serra}

Like other abstract artists, the architectural sculptures of Serra are intended to explore the relationship between the artwork and the viewer, the process of viewing not the process of representation. For Serra, it is the viewer whom is the subject. The scale, curves and torques of his work destabilize the viewer, deconstructing the horizontalvertical everyday context, rather like Mondriaan's lozenge paintings. Our IEs have placed 'Serra walls' within outdoor terrain and within a large gallery space. These curvilinear sheets and folds, (see Figure 9) define a circulation of viewers, guiding them into a goal-les movement. UnrealEd allows the direct construction of such walls, and circulation can be visualized through the programming of NPCs to explore the constrained space.

\section{James Turrell}

The American artist James Turrell (b.1943) takes art into the realm or pure perception. Gone are notions of mental representations of objects, scenes or ideas, the viewer is confronted with their process of viewing. In contrast to the Cubists and Expressionists engagement with depiction of a 'subject', here there is no subject: A purity of expression (and involvement) associated with the absence of a 'subject' (characterised by Rothko and Albers) is highlighted. The light which has been 
traditionally used to illuminate an associative or symbolic thought now becomes the focus of the viewer's experience. While light has been the tool of illumination, Turrell elevates light to an object of experience. Viewers see light as light, and so contemplate their own perceptual viewing. Light becomes visible; it is the message, not the messenger. In the visual ambiguities reated by Turrell's works, the viewer is invited to withdraw and to contemplate. Here is a distinction between Rothko and Newman who invited the viewer to engage. Turrell espouses Zen; he refers to his works as 'koans' and attempts to move those logical and philosophical paradoxes into the phenomenology of art. It is interesting that the UT affordances of lighting and color can be deployed to investigate and construct these high-art concepts.

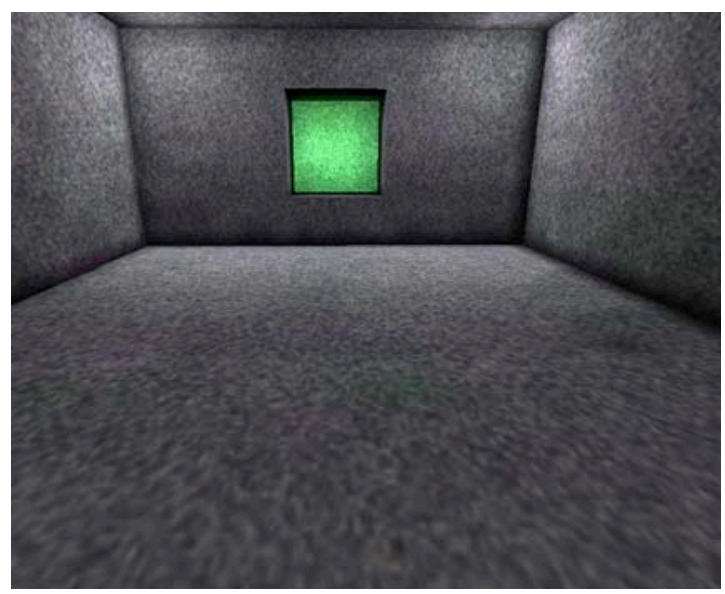

Figure 10. Turrell installation IE. The green square is not a canvas but a hole into a second room.

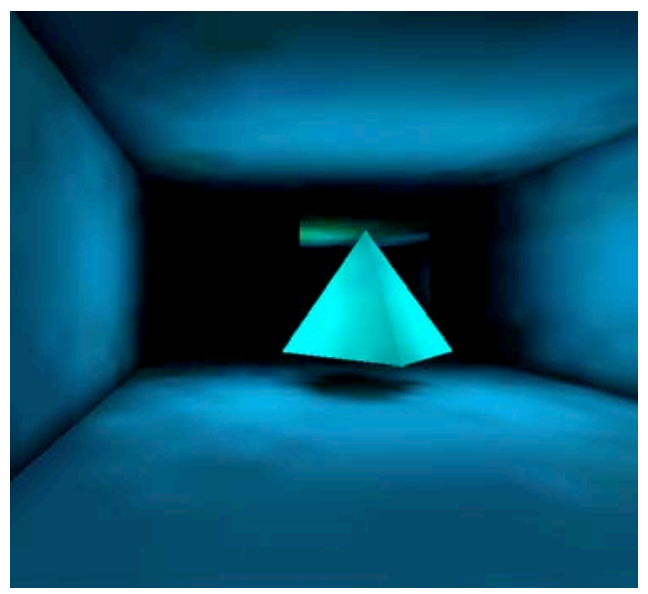

Figure 11. Turrell installation IE. The viewer may walk through the pyramid which appears as pure light.

Figure 10 reproduces a Turrell installation. Standing at the entrance to the exhibition room, the viewer perceives a canvas of green texture. However, on moving through the room, it becomes apparent that the green is pure light, emanating from a hole in the wall. In Figure 11, the apparently solid pyramid turns out to be a pure region of light, through which the viewer may pass unhindered.

\section{NEW CONCEPTS AND THEORIES OF ART}

\section{A Classification of Installation (Locative) Art}

We propose a classification of Installation Art according to the nature of the artwork's movement. (i) Static Installations. Here there is mo explicit movement of the installation elements. Any movement occurs indirectly, through the abstract work, or from the spatial movement of the viewers. (ii) Kinetic Installations where the elements are allowed to move in space. Their location can be defined using the 'movers' function within UnrealEd, or via Unreal Script programmes. (iii) Dynamic Installations where the dynamics of the state of the elements is allowed to change. The state of an element may typically comprise color, form, and size.

An example of a static installation is shown in Figure 12. Here the novelty is the scale of the installation. The viewer is able to walk and jump between the elements and 
to experience a sense of vastness. No longer must the installation be confined to the space of a gallery, it may occupy a city-space or indeed the cosmos.

Our approach to the design of kinetic and dynamic installations draws on the mathematical theory of 'Complex Dynamical Systems' (CDS). This is both an analytical and generative approach which has emerged to explain the nature and formation of spatial and temporal structures observed in nature. Examples include animal coat patterns and neural circuits responsible for animal gaits or swimming fish. The unifying element is the relational, how state, space and time are united in a formal (mathematical) language of relationships. These are incorporated into the IEs through programming in Unreal Script. As an example of a kinetic installation, we have realised one of Moholy-Nagy's studies in equilibrium and have programmed the elements to rotate according to a mathematical description of fish neurodynamics, (Figure 11). The resulting Installation is capricious; while the interactions between shapes are 'programmed' the behaviour of the whole installation is emergent and unpredictable. Yet the observer perceives a 'dynamic Gestalt' where coherence of movement is seen, yet without a sequence of pattern.

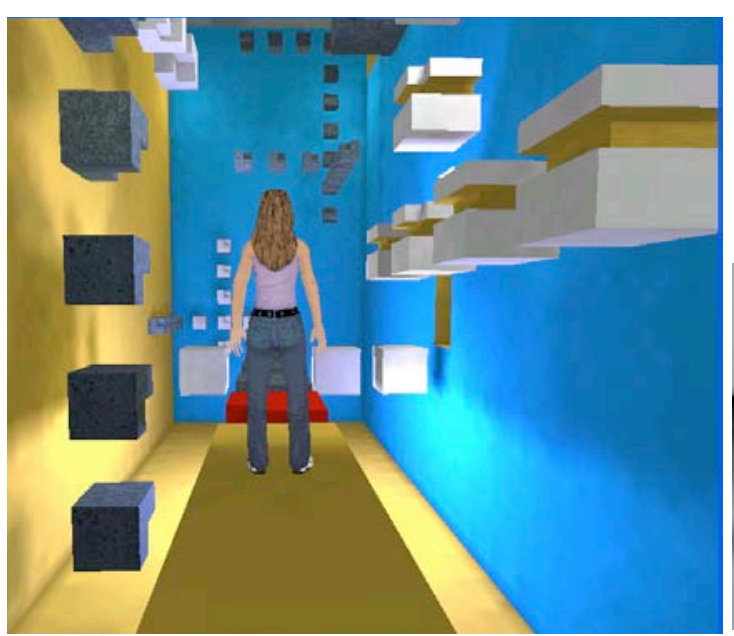

Figure 12. Static Installation which encompasses the spectator

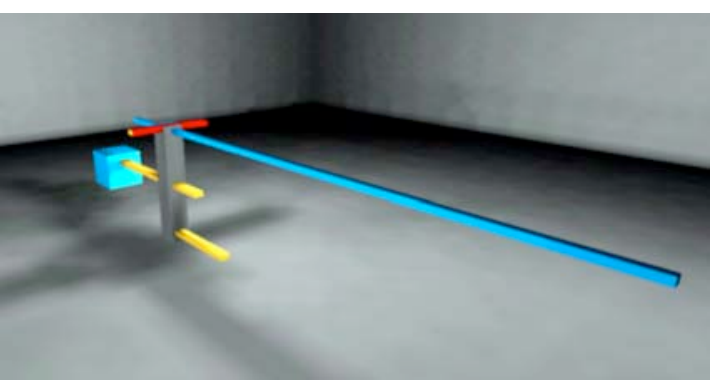

Figure 13. After Moholy-Nagy. Each element rotates interactively.

In a second Installation, we use the 'pixel boxes' of Bulloch which are programmed according to the collective behaviour of fireflies. Pulsating boxes of light become synchronized. This takes time; the viewer's indulgence is requested. Time devoted is rewarded by an experience of a visual fugue where layers of activity are experienced; dynamics of color, form and space. Hue is also rendered as the pitch of a sound chord.

\section{The Social perspective: Collaboration in experiencing Art.}

As Borriaud notes in his overview of the development of the function or art, we are now entering an epoch of artistic practise focussed on the establishment and exploration of inter-human relationships. "Any artwork might thus be defined as a relational object, like the geometrical place of a negotiation with countless correspondents and recipients [7]. It has always been the case that art needs a public and 
that public sensibilities change. This was recognized by Vygotsky in 1925 who asserted the social impact on the psychology of man and therefore his aesthetic feeling [8].

Our IEs defined by the UT2004 game engine are fundamentally collaborative. They allow many viewers to enter an IE, (equipped with headsets including microphones) and to have conversations while exploring the gallery or installation rooms. Our IEs allow the programming of 'Non-Player-Characters' (NPCs) in the IE. These may be scripted to have conversations with the viewers, providing a guide through an exhibition.

\section{REFLECTIONS AND CONCLUSIONS}

Through reflective consideration of the works of several twentieth-century artists, their approaches, philosophies and goals, combined with our creative experiences of working within a contemporary game technology, we strive to define a strategy for the development of a new medium in Art. This encompasses, but also extends, various Art movements and also generates some new. Our approach supports individual and collaborative experiences in both galleries and installations, and most significantly, suggests a new creative medium for artistic expression. Several common threads have emerged: (i) The notion of scale which is present in the static installations we propose, in the huge canvasses of Newman, and Riley's 'visual frequency'. This is explicit. Yet our IEs also support Delaunay's desire for 'vastness' and a movement to the 'sublime'. (ii) The notion of color which has been studied by Delaunay, Kandinsky and Klee, and realized in a perceptual-abstract form by Turrell. (iii) Movement is a concept common to much art, albeit explicit or implicit, from Kinetic art (the windy structures of Calder), the circulation of NPCs through Serra's confining plates to the implied movement of Klee and Malevich, and finally to the perceptual motion produced by Riley's works.

From the technological point of view, what can our IEs based on the UT2004 game engine technology provide for the creative artist? These are easily summarised: (i) The construction of a space, or gallery in which artworks may be experienced. Here, the scale and presence of this space may be realized. This space may be an enclosed room, or an external terrain, constructed to display sculptures or architectural buildings. Such a space may be defined by inhabitation (see Bachelard [9]) or by immersion (Rothko and Newman). It may consist of deformed geometrical surfaces to produce Riley's percept of movement. This space may be seen as an architectural space, defined by the circulation of its inhabitants. It may be constructed to either highlight or suppress the perspective view, it could contain realistic or abstract elements. (ii) It may contain static or dynamic geometric elements, painted with any texture which may be programmed to interact with the viewer. Such interaction may be made explicit through the use of rendered interaction triggers or implicit by concealing these triggers. (iii) Color, light and particle effects may be crafted to highlight phenomena or create the experience of new phenomena. (iv) Layers of sheets may be stacked; mirror surfaces can be introduced to create photorealistic effects such as in the work of Estes. (v) The game engine contains a 'physics engine' component which may be used to obtain kinetic effects, such as balls rolling around a 'Riley surface'. (vi) The game engine is essentially multi-player. Several viewers may enter the exhibition rooms with headphones and microphones and walk and talk together. This has potential use in establishing virtual galleries and museums. 
This technology can have a great impact on contemporary art, from the establishment of new spaces and modes of experience, to providing a new tool for the creative artist.

\section{ACKNOWLEDGEMENTS}

We acknowledge the work of June S. Moore and Laurence Wilde who have contributed to two IEs discussed in this paper.

\section{References}

[1] CHIPP, H.B: 1968, Theories of Modern Art, University of California Press. p317.

[2] idem, p319.

[5] BRESLIN, J.E.B: 1998, Mark Rothko, A Biography, Chicago University Press, p233.

[6] HESS, T.B: 1969, Barnett Newman, New York, p31.

[3] ELGAR, F: 1968, Mondrian, Thames and Hudson, London, p138.

[4] idem, p130.

[7] BOURRIAUD, N: 1998, Esthetique relationnelle (English Translation, Relational Aesthetics, 2002 Les presses du reel, p26).

[8] VYGOTSKY, L: 1925, The Psychology of Art, MIT Press (English Translation 1971).

[9] BACHELARD, G: 1994, The Poetics of Space, Beacon Press. 